\title{
Atypical Complications Following Surgical Excision of Carotid Body Tumors
}

\author{
Lauren H. Jain BSCH', Ian Fleetwood MD FRCSC ${ }^{2}$, Robert D. Hart MD FRCSC ${ }^{3}$, J.R. Trites MD FRCSC \\ S. Mark Taylor MD FRCSC ${ }^{3}$ \\ ${ }^{1}$ Class of 2013, Faculty of Medicine, Dalhousie University \\ ${ }^{2}$ Division of Neurosurgery, Department of Surgery, Dalhousie University \\ ${ }^{3}$ Division of Otolaryngology - Head and Neck Surgery, Department of Surgery, Dalhousie University
}

Paragangliomas are rare neuroendocrine tumors that account for $0.6 \%$ of all head and neck cancers; when they do arise, carotid body tumors constitute the majority (60\%). ${ }^{1,2}$ The carotid body is a small mass of tissue in the adventitia of each carotid bifurcation; they contain chemoreceptors, which monitor changes in arterial gas content and respond by mediating sympathetic flow, hence altering heart rate, blood pressure, and respiration rate. ${ }^{3}$ The majority of these tumors are sporadic (90\%), while the remainder are hereditary and more likely to be found bilaterally. ${ }^{4,5}$ The estimated incidence is 1 in 30,000, seen most commonly in middle-aged individuals, with a slight predilection for women. ${ }^{6,7,9} \mathrm{~A}$ well-recognized etiology of the sporadic type is chronic hypoxia; for example, carotid body tumors are seen more frequently in those living at high altitude and with chronic obstructive pulmonary disease. ${ }^{6,7}$

Carotid body tumors are typically asymptomatic and slow to proliferate; as a result, they may exist for years before an individual seeks medical attention. Most commonly, patients present with a hard, pulsatile, and typically painless mass in the upper neck. Once diagnosed, prompt surgical resection or removal of the tumor is recommended, as they can be locally aggressive. ${ }^{1}$ They can penetrate proximate spaces and compress select cranial nerves, resulting in numerous clinical symptoms. Additionally, carotid body tumors are generally benign, but they can develop malignant features, further necessitating the need for removal. ${ }^{8}$

The diagnosis and management of tumors in the parapharyngeal space is complex; the regional anatomy makes for a difficult, often delayed diagnosis as well as a challenging surgery. A multidisciplinary approach is essential to the management of these tumors, typically involving the expertise of radiation oncology, head and neck surgery, and neurosurgery. ${ }^{9}$ With advances in surgical techniques, blood replacement, and anesthetic management, the procedure is usually safe with low morbidity. ${ }^{10}$ However, like any surgical procedure, there are associated risks and potential complications. Of the post-surgical sequelae, some atypical syndromes have been observed. First-bite syndrome and auricular dystonia are rare complications following carotid body tumor excision; they serve as examples of complications seen subsequent to surgery in the parapharyngeal space. These specific atypical complications will be examined, with a focus on their unique etiologies and treatment. In the literature to date, reports of first-bite syndrome following carotid body tumor removal are of limited occurrence, and those on auricular spasm are scarce.

\section{Case 1}

A 45-year-old male presented to the Otolaryngology Head and Neck Surgery Clinic reporting sharp, intense pain in the parotid region upon eating. Approximately 3 months prior, he had undergone a left neck dissection to excise an $8 \mathrm{~cm}$ carotid body tumor. The patient had also had an internal carotid artery test occlusion and endovascular embolization prior to the procedure. The pain was first noticed while eating, and was described as a toothache in the soft tissues. The pain worsened when consuming acidic foods, and seemed to be related to any food being inserted into the mouth eliciting salivary stimulation. To relieve his symptoms, he was started on conservative measures including massage, warm compresses, and sialagogues. These proved to offer no relief of his symptoms, and injections of botulinum toxin type A (BTA), also known as Botox, were subsequently prescribed. 50 units were injected into the periparotid region and upper neck, specifically addressing his point of maximal tenderness. Injections are ongoing at 3-month intervals. Though his symptoms are not completely eliminated, he has noticed a remarkable improvement with this treatment. 


\section{Case 2}

A 30-year-old, otherwise healthy male presented to the Otolaryngology - Head and Neck Surgery Clinic reporting pronounced, ongoing left ear and neck muscle twitching following a left neck dissection to remove a $4 \mathrm{~cm}$ carotid body tumor. The spasms were described as both voluntary and involuntary. Physical examination revealed objective cervical dystonia in the distribution of the platysma as well as additional spasm of the auricularis posterior and superior. The patient was able to produce voluntary ipsilateral auricular twitching on command, but was unable to do so on the non-operated side. He reported being unable to elicit such activity prior to surgery. His facial and lower cranial nerve function were tested and found to be normal. Physiotherapy, including massage, stretching, and heat did not relieve his symptoms, and a trial of BTA injections was discussed but has yet to be pursued.

\section{Discussion}

The compact parapharyngeal space contains a plethora of vital structures. The vascularity of carotid body tumors, as well as their proximity to, and intimacy with, vital head and neck components necessitates a multidisciplinary approach. Treatment strategies include surgical excision, radiation therapy, and watchful waiting. ${ }^{9}$ Treatment selection is based on "the location and extent of the tumor, the presence of multiple tumors, the age and health of the patient, and the preferences of the patient and physician." ${ }^{11}$ In the literature, debate exists as to which modality is preferable. ${ }^{11}$ Surgical excision has been preferred, as it is the only available curative therapy; ${ }^{2,12}$ all carotid body tumors eventually become symptomatic and have the potential to undergo malignant transformation, which occurs in $10-15 \% .{ }^{5,78}$ However, excision is a potentially morbid procedure, frequently associated with excess damage to nearby vascular and neural structures. ${ }^{11}$

Radiotherapy is another option, though it has debated as a primary treatment modality as carotid body tumors were historically considered to be radio-resistant. ${ }^{13}$ These claims have been criticized however, as they are thought to have been based on limited studies where only "large, recurrent or inoperable tumors" were treated by radiation therapy. ${ }^{7}$ More recent studies have demonstrated external beam radiation to be well tolerated and show good responses, with control rates of $90 \%-98 \%$ at 5 years. ${ }^{7-9,13}$ Total resolution is rare, though in those with an asymptomatic mass following radiation, quiescent tumors are equated to being cured. ${ }^{5,14}$ Currently, surgical excision is typically recommended for small resectable tumors in patients who are "medically operable and in whom surgery would not require the sacrifice of major neural and/or vascular structures, and cause excessive morbidity."11 Indications for external beam radiation are typically reserved for unresectable or recurrent tumors, tumors that have metastasized to regional lymph nodes, and poor surgical candidates. ${ }^{7-9,13}$ Watchful waiting is another option that should be considered in asymptomatic individuals, or those with an otherwise limited life expectancy. ${ }^{9}$ When tumor resection is indicated, surgical expertise is essential for the successful excision of a carotid body tumor and the minimization of post-surgical complications, some of which may be inevitable. Cases vary considerably, and complications are often dependent upon the size, location, and the degree of vascularity of the tumor.

The Shamblin classification system is useful in anticipating the degree of surgical difficulty and postoperative complications, based on the relationship of tumors to the carotid vessels. Class I tumors can be separated easily from the vessel wall, while Class II tumors are more involved, partially surrounding the carotid artery necessitating delicate sub-adventitial dissection to prevent arterial injury. ${ }^{10}$ The greatest complication risk, approximately $67 \%$, is seen with Shamblin Class III tumors, which are larger than 5 $\mathrm{cm}$ in diameter, and adhere to and completely encircle the carotid vessels. ${ }^{1,3}$ Dissection is difficult and carotid replacement may be required.

Of the post-surgical sequelae, cranial nerve damage is the most frequently observed, arising in over $15 \%$ of cases; the glossopharyngeal, vagus, spinal accessory, and hypoglossal nerves are the most likely to be affected. ${ }^{8}$ As a result, patients may display cranial nerve palsies, tongue deviation, or a shoulder drop, and experience progressive pain, hoarseness, dysphagia, or odynophagia. ${ }^{1}$ Depending on the degree of damage and the specific structures involved, significant post-operative morbidity can result. Baroreceptor failure is often seen following bilateral tumor resection, as well as transient ischemic attacks and strokes. Additionally, the sympathetic chain can be damaged during resection, resulting in Horner's syndrome. ${ }^{10}$ Occasionally, there are atypical complications following carotid body tumor excisions, with first-bite syndrome being a prime example. Understanding the specific etiology and appropriate treatments is essential for minimizing post-operative morbidity of this condition.

\section{First-Bite Syndrome}

First-bite syndrome is a rare complication seen in patients following surgical procedures in the parapharyngeal space. The literature reporting first- 
bite syndrome following carotid body tumor excision is limited, with few cases having been described to date, which makes this case of particular interest. It is likely overlooked and underreported, as it is a minor complication, though its impact on patient quality of life makes it an important topic of study. Firstbite syndrome is characterized by a sharp, intense, ipsilateral pain in the parotid region upon taking the initial bite of a meal. The pain is due to powerful spasm or cramping in the vicinity of the parotid gland. ${ }^{15}$ It can also cause "intense ear pain with strong sialagogues," saliva inducing agents such as tart or bitter foods. ${ }^{16}$ The pain gradually lessens with subsequent mastication, but is nonetheless as painful upon taking the first bite of the next meal. ${ }^{15}$ The symptoms of the patient presented in Case 1 are remarkably analogous to the description of this syndrome. Although the pathogenesis of first-bite syndrome has yet to be confirmed, research findings to date have been consistent with the hypothesis put forth by Netterville et al, who first described the syndrome as a complication following resection of vagal paragangliomas. The authors hypothesized that first-bite syndrome resulted from a loss of sympathetic innervation to the parotid gland. ${ }^{17}$

A retrospective chart review by Chiu et al examined the common features of 12 patients that had developed first-bite syndrome subsequent to operations of the parapharyngeal space. ${ }^{18}$ The objective was to confirm the etiology of first-bite syndrome as a parotid gland sympathectomy. In the study, 6 patients had undergone resection of the sympathetic chain, and the external carotid artery had been ligated inferior to the parotid gland in the remaining 6 patients. ${ }^{16}$ All cases seem to suggest interrupted sympathetic innervation as the underlying cause of first-bite syndrome in these patients. The superior cervical ganglion feeds the external carotid artery plexus, which proceeds to innervate the parotid gland. The parotid gland is dually innervated (Fig. 1), also receiving parasympathetic innervation from the glossopharyngeal nerve via the otic ganglion. ${ }^{15}$ Once in the parotid substance, both autonomic nerves travel to their destination within the same Schwann-axon bundle and cause myoepithelial cell contraction upon stimulation. Thus, upon oral intake and subsequent biting and chewing, parasympathetic neurotransmitters are released, which cross-stimulate the sympathetic receptors of the parotid gland. This creates a supramaximal myoepithelial cell response, or excessive contraction resulting in pain that can be incapacitating. ${ }^{17}$ In this study, one patient had experienced first-bite syndrome following the excision of a carotid body tumor, strengthening the evidence that the etiology of first-bite syndrome in the patient presented in Case 1 was identical; the substantial tumor size, and the close anatomical relation of the aforementioned sympathetic inputs to the bifurcation of the carotid artery makes sympathetic nerve damage a likely explanation. Another notable case of post-operative first-bite syndrome has been reported by Casserly et al, where a cervical sympathetic chain schwannoma (CSCS) was misdiagnosed as a carotid body tumor. ${ }^{19}$ This is of relevance as it is among the few reported cases of a tumor in the precise region of the carotid bifurcation that resulted in first-bite syndrome, therefore aiding in the further elucidation of the etiology.

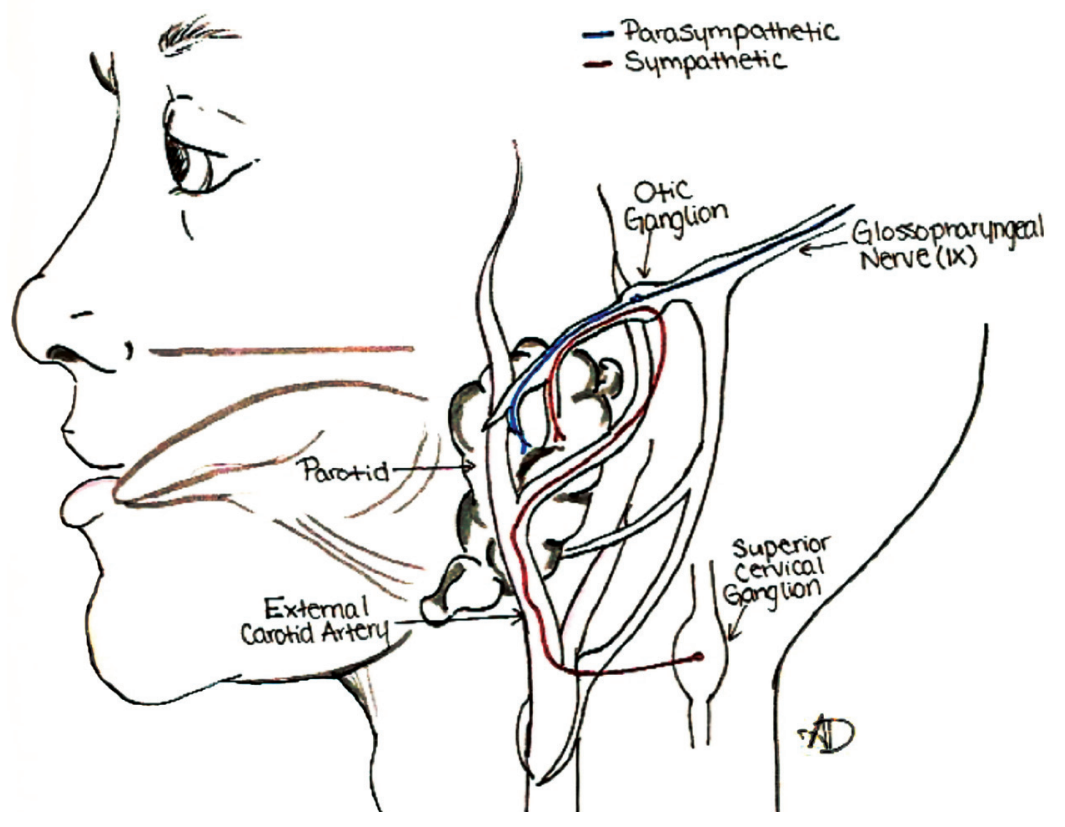

Figure 1. The anatomic relationship between the sympathetic and parasympathetic innervation to the parotid gland. In first-bite syndrome, the sympathetic innervation has been interrupted following surgery in the parapharyngeal space. Upon taking the first bite of a meal, which induces salivation, the parasympathetic neurotransmitters cross-stimulate the sympathetic receptors in the parotid gland, causing intense pain. (Illustration by Alyson Digby, Dalhousie Medicine Class of 2016). 
Upon external examination, carotid body tumors present remarkably similar to cervical sympathetic chain schwannomas, "benign neoplasms from the myelin-producing Schwann cells of the nervous sheath." ${ }^{19}$ They too are slow growing, painless tumors; they exhibit the same external presentation as carotid body tumors, and are found in the same vicinity within the neck. They result in a similar splaying of the internal and external carotid arteries, known as the "Lyre sign," and even with radiological preoperative assessment, distinguishing the two tumor types proves difficult. ${ }^{19}$ In this case, the tumor types were mistaken, and re-diagnosed intra-operatively. In the surgical resection of this schwannoma, which was found on the sympathetic chain itself, resection of the nerve was inevitable, resulting in the subsequent loss of sympathetic input to the parotid gland, manifesting as intense pain by the same mechanism as outlined above. Based on these findings, other tumors excised from the immediate vicinity of carotid body tumors have resulted in first-bite syndrome, further suggesting sympathetic damage as the probable etiology in our patient.

To date, few successful treatments for this syndrome have been found. Non-steroidal anti-inflammatory drugs and carbamazepine have been attempted to treat the symptoms, but to no avail. Surgical interventions have also proven unsuccessful. ${ }^{15}$ Our patient was treated with BTA injections at 3-month intervals, and demonstrated significant improvement. BTA works by blocking the transmission of parasympathetic impulses, specifically preventing the release of acetylcholine from the pre-synaptic neuron to various muscle and glandular tissues..$^{20}$ This translates into a reduction of pathological glandular secretion and movement. Due to its efficacy, minimally invasive nature, and the rarity of side effects, it has become a mainstay treatment for numerous disorders of the head and neck region. The ensuing reduction in pain associated with first-bite syndrome is a promising development for patients presenting with this atypical, debilitating complication.

\section{Auricular Dystonia}

Auricular spasm following the excision of a carotid body tumor is another abnormal post-surgical sequela for which the exact mechanism of development remains unclear. However, the surgical history and clinical findings of the patient presented in Case 2 make it a likely instance of facial synkinesis, "an involuntary movement accompanying a voluntary one." ${ }^{22}$ Hypotheses for this manifestation can therefore be proposed based on the existing literature on facial synkinesis. The mechanism for the development of this phenomenon is thought to be the abnormal, undifferentiated rewiring of the facial nerve (CNVII) following trauma; in those with unilateral paralysis, the development of facial synkinesis parallels the timing of the return of motor function. This aberrant regeneration of the damaged nerve to fibers other than the original muscles is referred to as "sprouting." 22 Depending on the case series, synkinesis has been estimated to occur in $15-50 \%$ of individuals following facial nerve paralysis. ${ }^{21,22}$ Husseman and Metha proposed possible mechanisms of synkinesis and, in addition to misdirected regeneration, suggest it may involve "neighboring axons stimulating each other or a hyper excitability of the facial nucleus."22

Patients experience abnormal, synchronous, involuntary facial muscle movements in conjunction with the voluntary movement of another facial muscle group. ${ }^{21,22}$ This movement is often elicited with various emotional facial expressions. ${ }^{23}$ The disorder can impart significant distress on an individual, as it can affect eating, drinking, and speaking. In addition, it may hinder the ability to display appropriate facial expressions, resulting in embarrassment, poor self-esteem and consequently impairing quality of life. ${ }^{22,25}$

Synkinesis is frequently observed in the platysma, though facial nerve rerouting to the auricularis muscles, the extrinsic muscles of the ear, is a novel case. These muscles are innervated by the temporal and posterior auricular branches of the facial nerve. The facial nerve exits the skull through the stylomastoid foramen, passes laterally to the external carotid artery, and pierces the parotid gland to then innervate the numerous muscles of facial expression. ${ }^{24}$ Before the nerve enters the parotid gland, branches supplying the posterior digastric, stylohyoid and posterior auricular muscles are given off. The structures of the region are evidently intimately related, thus it is plausible that removal of the carotid body tumor may have involved some degree of trauma to the facial nerve; its defective regeneration may have subsequently resulted in aberrant innervation of a nearby muscle. Though the lower cranial nerves are most commonly compromised in carotid body tumor removal, this may be a rare instance of facial nerve involvement.

In reports by Laskawi and Filipo et al, treatment with BTA demonstrated notable efficacy in reducing such abnormal and distressing synkinetic movement disorders in the head and neck. ${ }^{23,25}$ As mentioned previously, BTA prevents the transmission of autonomous impulses to various muscle and glandular tissues causing a "functional denervation of neuromuscular end plates." ${ }^{25}$ Subsequent pathological movement to the targeted region is halted, thereby 
improving one's quality of life. ${ }^{20}$ Such evidence makes BTA a promising treatment option for the patient presented in Case 2, and other patients experiencing similar post-surgical facial spasms.

\section{Conclusion}

The parapharyngeal space is a compact, highly vascular territory containing countless vital structures. Its complex anatomy necessitates a careful, practiced surgical approach. For surgeons operating in the parapharyngeal space, an awareness and understanding of the pathophysiology of rare post-operative complications may aid in minimizing their occurrence; first-bite syndrome and auricular dystonia following carotid body tumor excision are examples of potential complications following removal of parapharyngeal tumors. Evidence supports the interruption of sympathetic innervation to the parotid gland as the causative factor in first-bite syndrome development, while facial nerve synkinesis is a likely explanation for the display of auricular dystonia. Importantly, prevention of these complications may be unavoidable due to the vascularity and involvement of the tumor. Thus, an awareness of these complications is essential to guide pre-operative counseling and hasten post-operative recognition. In addition, knowledge of the most current and efficacious treatment options is essential for mediating such complications when they do arise. BTA, a safe and minimally invasive therapeutic option, has been used extensively for treating head and neck disorders, of variable etiologies. It has been shown to minimize symptoms of first-bite syndrome and facial dystonia, thus ameliorating the quality of life of these patients. It is a promising therapeutic option when similar cases arise.

\section{References}

1. Georgiadis G, Lazarides M, Tsalkidis A, et al. Carotid body tumor in a 13-year-old child: case report and review of the literature. Journal of Vascular Surgery 2008; 47(4): 874-880.e3.

2. Albsoul N, Alsmady M, Al-Aardah M, Altaher R. Carotid body paraganglioma management and outcome. European Journal of Scientific Research 2009; 37(4): (567-574).

3. Tinkham MR. Care of the patient undergoing surgical excision of a carotid body chemodectoma. AORN Journal 2010; 91(1):117-131.

4. Ridge B, Brewster D, Darling R, Cambria R, LaMuraglia G and Abbott W. Familial carotid body tumors: incidence and implications. Annals of Vascular Surgery 1993; 7(2): 190-194.

5. Hinerman R, Amdur R, Morris C, Kirwan J, Mendenhall W. Definitive radiotherapy in the management of paragangliomas arising in the head and neck: a 35-year experience. HEAD \& NECK 2008; 1431-1438.

6. Sajid M, Hamilton G, Baker D. A multicenter review of carotid body tumour management. Eur J Vasc Endovasc Surg 2007; 34:127-130.
7. Singh D, Pinjala R, Reddy R, Vani P. Management for carotid body paragangliomas. Interactive CardioVascular and Thoracic Surgery 2006; 5: 692-629.

8. Makeieff M, Raingeard I, Alric P, et al. Surgical management of carotid body tumors. Annals of Surgical Oncology 2008: 15(8): 2180-2186.

9. Chino J, Sampson J, Tucci D, Brizel D, and Kirkpatrick J. Paraganglioma of the head and neck: long-term local control with radiotherapy. American Journal of Clinical Oncology 2009; 32(3): 304-307.

10. Lees CD, Levine HL, Beven EG, et al. Tumors of the carotid body. The American Journal of Surgery 1981; 142:362-365.

11. Netterville J, Reilly K, Robertson D, Reiber M, Armstrong W, Childs P. Carotid Body Tumors: A Review of 30 Patients with 46 Tumors. Laryngoscope 1995; 105 (115-126).

12. Tayyab M, Khan M, Sophie Z. Presentation and management of carotid body tumors. Journal of Pakistan Medical Association 2003; 53(7).

13. Davidovic L, Djukic V, Vasic D, Sindjelic R and Duvnjak S. Diagnosis and treatment of carotid body paraganglioma: 21 years of experience at a clinical center of Serbia. World Journal of Surgical Oncology 2005; 3(10).

14. Knight T, Gonzalez J, Rary J, Rush D. Current concepts for the surgical management of carotid body tumor. The American Journal of Surgery 2006; 191(104-110).

15. Kawashima Y, Sumi, T, Sugimoto T, et al. First-bite syndrome: A review of 29 patients with parapharyngeal space tumor. Auris Nasus Larynx 2008; 35:109-113.

16. Kamal A, El-Fattah AM, Tawfik A, Razek A. Cervical sympathetic schwannoma with postoperative first bite syndrome. Eur Arch Otorhinolaryngol 2007; 264:1109-1111.

17. Netterville J, Jackson C, Miller F, Wanamaker J, Glasscock M. Vagal paraganglioma a review of 46 patients treated during a 20year period. Otolaryngol Head and Neck Surg 1998; 124:11331140.

18. Chiu A, Cohen J, Burningham A, et al. First bite syndrome: A complication of surgery involving the parapharyngeal space. Head \& Neck 2002:996-999.

19. Casserly P, Kiely P, Fenton J. Cervical sympathetic chain schwannoma masquerading as a carotid body tumour with a postoperative complication of First-bite syndrome. Eur Arch Otorhinolaryngol 2009; 266:1659-1662.

20. Lee B, Lee J, Lee $Y$, et al. Novel treatment of first bite syndrome using Botulinum Toxin Type A. HEAD \& NECK 2009; 31(8):989993.

21. Mehta R, Wernick Robinson M, Hadlock TA. Validation of the Synkinesis Assessment Questionnaire. The Laryngoscope 2007; 117:923-926.

22. Beurskens C, Oosterhof J, Nijhuis-van der Sanden M. Frequency and location of synkineses in patients with peripheral facial nerve paresis. Otology \& Neurotology 2010; 31:671-675.

23. Laskawi R. The use of botulinum toxin in head and face medicine: An interdisciplinary field. Head \& Face Medicine 2008; 4(5).

24. Kosins A, Hurvitz K, Evans G, et al. Facial paralysis for the plastic surgeon. Can J Plast Surg 2007;15(2):77-82.

25. Filipo R, Spahiu I, Covelli E, Nicastri M, Bertoli G. Botulinum toxin in the treatment of facial synkinesis and hyperkinesis. The Laryngoscope 2012; 122:266-270. 